\title{
Digital gene expression analysis of gene expression differences within Brassica diploids and allopolyploids
}

\author{
Jinjin Jiang, Yue Wang, Bao Zhu, Tingting Fang, Yujie Fang and Youping Wang*
}

\begin{abstract}
Background: Brassica includes many successfully cultivated crop species of polyploid origin, either by ancestral genome triplication or by hybridization between two diploid progenitors, displaying complex repetitive sequences and transposons. The U's triangle, which consists of three diploids and three amphidiploids, is optimal for the analysis of complicated genomes after polyploidization. Next-generation sequencing enables the transcriptome profiling of polyploids on a global scale.
\end{abstract}

Results: We examined the gene expression patterns of three diploids (Brassica rapa, B. nigra, and B. oleracea) and three amphidiploids (B. napus, B. juncea, and B. carinata) via digital gene expression analysis. In total, the libraries generated between 5.7 and 6.1 million raw reads, and the clean tags of each library were mapped to 18547-21995 genes of $B$. rapa genome. The unambiguous tag-mapped genes in the libraries were compared. Moreover, the majority of differentially expressed genes (DEGs) were explored among diploids as well as between diploids and amphidiploids. Gene ontological analysis was performed to functionally categorize these DEGs into different classes. The Kyoto Encyclopedia of Genes and Genomes analysis was performed to assign these DEGs into approximately 120 pathways, among which the metabolic pathway, biosynthesis of secondary metabolites, and peroxisomal pathway were enriched. The non-additive genes in Brassica amphidiploids were analyzed, and the results indicated that orthologous genes in polyploids are frequently expressed in a non-additive pattern. Methyltransferase genes showed differential expression pattern in Brassica species.

Conclusion: Our results provided an understanding of the transcriptome complexity of natural Brassica species. The gene expression changes in diploids and allopolyploids may help elucidate the morphological and physiological differences among Brassica species.

Keywords: Brassica spp, Polyploidization, Sequencing, Digital gene expression (DGE)

\section{Background}

Polyploidy is an important factor in the evolution of many plants and has attracted considerable scientific attention for a long period of time. Many important economical crops are of polyploid origin, including wheat, cotton, and rapeseed [1]. Cruciferae includes the model species Arabidopsis thaliana and the economically important Brassica crops. These important crops include three diploid Brassica species, namely, B. rapa (AA, $2 n$ = 20; Chinese cabbage, turnip, turnip rape), B. nigra (BB,

\footnotetext{
* Correspondence: wangyp@yzu.edu.cn

Jiangsu Provincial Key Laboratory of Crop Genetics and Physiology, Yangzhou University, Yangzhou 225009, China
}

$2 n=16$; black mustard), and B. oleracea (CC, $2 n=18$; cauliflower, broccoli, kale), and three allopolyploids spontaneously derived from pairwise hybridization of the diploids, which are $B$. napus (AACC, $2 n=38$; oilseed rape, swede), $B$. juncea (AABB, $2 n=36$; abyssinian or Ethiopian mustard), and B. carinata (BBCC, $2 n=34$; Indian or brown mustard) [2]. Lysak et al. (2005) confirmed the chromosome triplication history of Brassica that corresponds to that of Arabidopsis [3]. Cheung et al. (2009) found that the divergence of Arabidopsis and Brassica lineage was approximately $17 \mathrm{Mya}$ [4], and the replicated Brassica subgenomes (probably the divergence of $\mathrm{A} / \mathrm{C}$ from $\mathrm{B}$ genome) was diverged by $14.3 \mathrm{Mya}$ [4]. In addition, the $\mathrm{A}$ and $\mathrm{C}$ genomes were estimated 
with 3.7 Mya divergence. Woodhouse et al. (2014) stated that the $B$. rapa lineage underwent a whole-genome triplication of 5-9 Mya [5]. For the allopolyploids, B. napus probably arose from the natural hybridization of $\mathrm{A}$ and C genomes around 10,000 years ago. However, when the hybridization between $\mathrm{A}$ and $\mathrm{B}$ genomes and between $\mathrm{B}$ and $C$ genomes happened is still unclear. The precise ancestors of $B$. napus, B. juncea, and B. carinata are not yet identified [6]. The duplication of gene segments reported on Brassica is explained as frequent loss, remote genome duplication, or unbalanced homologous recombination [7]. During the divergence of Brassica species, the sub-functionalization and/or neo-functionalization of these paralogs coupled with novel gene interactions contribute significantly to genome evolution [8]. Moreover, genetic mapping and sequencing analysis substantiate the triplication hypothesis of diploid Brassica genomes [9-12]. The comparative mapping of Brassica by using genetic markers has successfully revealed homologous rearrangements, translocations, and fusions that are crucial to the diversification of the $\mathrm{A}, \mathrm{B}$, and $\mathrm{C}$ genomes from A. thaliana [13-15].

Many linkage maps and karyotype analysis have identified extensive collinearity and genomic polymorphisms among Brassica genomes. Given the complexity of the gene copy number and syntenic conservation caused by polyploidization, Brassica genomes are difficult to study $[16,17]$. Identifying the genes related to specific traits based on the linkage maps is also challenging because of the complexity of the homologs and paralogs in polyploidy genomes $[15,18]$. Profiling arrays of $A$. thaliana are useful in the transcriptome analysis of Brassica [6]. However, A. thaliana-based microarrays lack the resolution of Brassica specific genes and paralogous genes. Furthermore, Brassica microarrays were developed to confirm Brassica-specific expressed genes [19]. Identifying different homologous copies of Brassica sequences is challenging for microarray expression analysis [20]. Next-generation sequencing is an optimal method for genomic and transcriptomic studies and provides opportunities for polyploidy studies and enables the extensive genome profiling of crops with complex genomes, such as soybean, potato, tomato, cotton, maize, and common bean [21-26]. This technology also promotes sequencing analysis in Brassica; the genome sequence of B. rapa has already been released and annotated [12]. The genome sequencing of $B$. oleracea, $B$. nigra, and $B$. napus is still in progress. However, the genome sequences of B. oleracea are available in the Basic Local Alignment Search Tool in Brassica database (www.brassica.info). The transcriptome profiling of B. napus has been analyzed via RNA sequencing [27-29]. This information is valuable for the investigation of Brassica genome evolution. Many technologies have been applied to quantify transcript abundance, including microarray, serial analysis of gene expression, digital gene expression (DGE), and RNA-seq. DGE and RNA-seq have been widely used to identify the molecular information of plant transcriptome and gene expression variation between comparable samples. DGE, as a well-known technique suitable to directly quantify transcript abundance counts, is optimized over RNA-seq because of its cost efficiency. RNAseq is a flexible approach that can detect full-transcript sequence, alternative splicing, exon boundaries, and transcript abundance. However, each transcript in RNAseq can be mapped multiple times, and the sequencing depth of RNA-seq is correlated with but is not equal to transcript abundance. Each read in DGE is expected with a sole hit on an RNA molecule. Therefore, DGE is better to represent rare transcripts or exclude transcripts with less interest than RNA-seq [30].

Many studies have analyzed the genomic and phenotypic changes in synthesized Brassica, particularly B. napus and hexaploid Brassica [31-33]. However, limited information is available for the natural species of Brassica. In the present research, we performed DGE analysis on three diploid Brassica species (B. rapa, B. nigra, and B. oleracea) and three allopolyploids (B. napus, $B$. juncea, and $B$. carinata) to determine the transcriptome changes after natural polyploidization. The expression profile of the genes in the six Brassica species was reported, and the multiple gene expression differences were observed. Differentially expressed genes (DEGs) are involved in a wide range of stress resistance and development processes. This study is the first transcriptomic research that identifies DEGs and the pathways involved in the natural polyploidization of the six Brassica species.

\section{Results}

\section{DGE profile}

This research investigates the transcriptome profiling of diploids and spontaneous allopolyploids in Brassica by performing DGE analysis on the seeding stage of the six Brassica species, namely, B. rapa $(\mathrm{Br}), B$. nigra $(\mathrm{Bg})$, B. oleracea (Bo), B. napus (Bn), B. juncea (Bj), and B. carinata $(\mathrm{BC})$. DGE libraries from the leaves of four-week-old plants were generated and sequenced by an Illumina technology. The sequence data are available from the GEO repository with an accession number of GSE43246. The statistics of the DGE tags are shown in Table 1. Approximately six million raw tags were generated for each library. Clean tags were obtained after removing the low-quality sequences and adaptor sequences from the raw data. 6178564, 5881618, 6059222, 5964594, 6076830, and 5795234 clean tags were obtained in $\mathrm{Br}, \mathrm{Bg}, \mathrm{Bo}, \mathrm{Bn}, \mathrm{Bj}$, and $\mathrm{Bc}$, respectively. Unambiguous tags (tags that were uniquely matched to one gene of reference genome with no more than one mismatch) were counted and normalized to TPM 
Table 1 Statistics of categorization and abundance of DGE tags

\begin{tabular}{|c|c|c|c|c|c|c|c|}
\hline Summary & & B. rapa & B. nigra & B. oleracea & B. napus & B. juncea & B. carinata \\
\hline Raw Tag & Total & 6178564 & 5881618 & 6059222 & 5964594 & 6076830 & 5795234 \\
\hline Raw Tag & Distinct Tag & 293575 & 214427 & 243895 & 269285 & 400134 & 278768 \\
\hline Clean Tag & Total number & 6018254 & 5772449 & 5930726 & 5823113 & 5858527 & 5657697 \\
\hline Clean Tag & Distinct Tag number & 133499 & 106552 & 116771 & 128967 & 181965 & 142281 \\
\hline Tag Mapping to Gene & Total number & 1964909 & 1990442 & 1747843 & 2253347 & 1857572 & 1915305 \\
\hline Tag Mapping to Gene & Distinct Tag number & 44267 & 30413 & 36220 & 45358 & 56289 & 40425 \\
\hline Unambiguous Tag Mapping to Gene & Total number & 1679848 & 1635594 & 1475050 & 1924944 & 1531974 & 1594991 \\
\hline Unambiguous Tag Mapping to Gene & Total\% of clean tag & $27.91 \%$ & $28.33 \%$ & $24.87 \%$ & $33.06 \%$ & $26.15 \%$ & $28.19 \%$ \\
\hline Unambiguous Tag Mapping to Gene & Distinct Tag number & 39414 & 26114 & 31933 & 40561 & 49892 & 35285 \\
\hline Unambiguous Tag Mapping to Gene & Distinct Tag\% of clean tag & $29.52 \%$ & $24.51 \%$ & $27.35 \%$ & $31.45 \%$ & $27.42 \%$ & $24.80 \%$ \\
\hline Tag-mapped Genes & number & 19023 & 16687 & 18547 & 19955 & 21995 & 19436 \\
\hline Tag-mapped Genes & $\%$ of ref genes & $46.20 \%$ & $40.53 \%$ & $45.05 \%$ & $48.47 \%$ & $53.42 \%$ & $47.20 \%$ \\
\hline Unambiguous Tag-mapped Genes & number & 16574 & 13867 & 15970 & 17448 & 19424 & 16645 \\
\hline Unambiguous Tag-mapped Genes & $\%$ of ref genes & $40.25 \%$ & $33.68 \%$ & $38.79 \%$ & $42.38 \%$ & $47.18 \%$ & $40.43 \%$ \\
\hline Mapping to Genome & Total number & 2437918 & 1147106 & 2105332 & 2164464 & 2047451 & 1462061 \\
\hline Mapping to Genome & Total\% of clean tag & $40.51 \%$ & $19.87 \%$ & $35.50 \%$ & $37.17 \%$ & $34.95 \%$ & $25.84 \%$ \\
\hline Mapping to Genome & Distinct Tag number & 44076 & 15159 & 30703 & 40689 & 50304 & 29547 \\
\hline Mapping to Genome & Distinct Tag\% of clean tag & $33.02 \%$ & $14.23 \%$ & $26.29 \%$ & $31.55 \%$ & $27.64 \%$ & $20.77 \%$ \\
\hline Unknown Tag & Total number & 1615427 & 2634901 & 2077551 & 1405302 & 1953504 & 2280331 \\
\hline Unknown Tag & Total\% of clean tag & $26.84 \%$ & $45.65 \%$ & $35.03 \%$ & $24.13 \%$ & $33.34 \%$ & $40.30 \%$ \\
\hline Unknown Tag & Distinct Tag number & 45156 & 60980 & 49848 & 42920 & 75372 & 72309 \\
\hline Unknown Tag & Distinct Tag\% of clean tag & $33.82 \%$ & $57.23 \%$ & $42.69 \%$ & $33.28 \%$ & $41.42 \%$ & $50.82 \%$ \\
\hline
\end{tabular}

Clean tags are tags after filtering low-quality tags from raw data. Distinct tags are different tags and unambiguous tags are the remaining clean tags after removing tags mapped to more than one locus of reference genome.

to evaluate the gene expression level. To evaluate the normality of the DGE data, the distribution of the total tags and distinct clean tags (tags with specific nucleotide sequence) over different tag copy numbers was analyzed. The distribution of the tag expression was similar for each library. Moreover, the distribution of clean tags in the six libraries showed that most of the tags are from highly expressed genes (Figure 1, Additional files 1 and 2). The percentage of distinct tags with high counts dropped dramatically, and the distinct tags with more than 100 copies accounted for less than $8 \%$. However, more than $67 \%$ of the total clean tags accounted for more than 100 copies in each library. By contrast, more than $43 \%$ of the distinct clean tags had copy numbers between two and five, which represented approximately $96 \%$ of the total number of clean tags. Generally, a small number of categories of mRNA showed high abundance, whereas the other majority had a quite low expression level. This finding indicates that only a small number of mRNAs are expressed at high abundance, and majority of them are expressed at very low levels [34].

The clean tags were then mapped onto the $B$ rapa genome with a maximum of one base-pair mismatch
[12]. Table 1 shows that the $1964909,1990442,1747843$, 2253347, 1857572, and 1915305 tags in Br, Bg, Bo, Bn, $\mathrm{B} \mathbf{j}$, and $\mathrm{BC}$ were mapped to $\mathrm{B}$. rapa genome, respectively. Statistical analysis of clean tag alignment was conducted, including the analysis of total clean tags and distinct clean tags (Additional files 2 and 3). More than $54 \%$ of the total clean tags and $42 \%$ of the distinct clean tags in each library were mapped onto the $B$. rapa genome. However, the tags mapped in the DGE library of Bg and $\mathrm{Bc}$ were lower than those in the other four libraries, which might be due to the divergence of the $\mathrm{B}$ genome to the A/C genome. Moreover, the tag mapping onto the B. rapa genome generated 19023 tag-mapped genes for Br, 16687 for Bg, 18547 for Bo, 19955 for Bn, 21995 for $\mathrm{Bj}$, and 19436 for Bc. In total, approximately $61 \%$ of the genes in the B. rapa genome (25298 genes) could be mapped with unique tags (Additional file 4). Furthermore, we mapped all the clean tags of each DGE library to the genome of $A$. thaliana, and the summary and details of the mapping result are listed in Additional file 5. Only approximately $47 \%$ of $A$. thaliana genes (19557 genes) were successfully mapped, and the percent of unambiguous tag-mapped genes in A. thaliana is much 


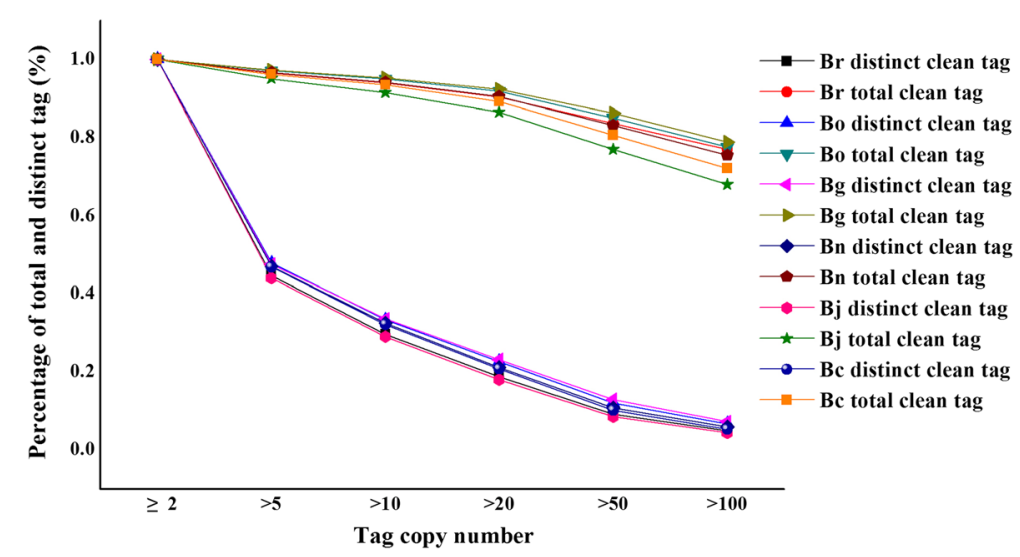

Figure 1 Distribution of total tag and distinct tag counts over different tag abundance categories from the six libraries.

lower than in B. rapa. The number of DGE tags in each library that well matched with Arabidopsis genome is also lower than that mapped to $B$. rapa. The difference in mapping rate is in accordance with the prediction that the A, B, and $\mathrm{C}$ genomes of Brassica diverged after the divergence of Arabidopsis and Brassica lineages [6]. Thus, we chose the mapping information that used $B$. rapa as reference for further analysis. Saturation analysis was performed to check if the number of detected genes increased with sequencing amount. The result showed that the number of detected genes stopped increasing when the number of reads reached 2 million (Additional file 6). The distribution of the ratio of distinct tag copy numbers in each pair of libraries was analyzed. More than $90 \%$ of the distinct tags had ratios up to five folds (Additional file 7).

\section{DEGs in Brassica diploids}

The DEGs in Brassica diploids (Br, Bg, and $\mathrm{Bo}$ ) were compared ( $\mathrm{Br}$ vs. $\mathrm{Bo}, \mathrm{Bg}$ vs. $\mathrm{Br}, \mathrm{Bg}$ vs. Bo, where $\mathrm{A}$ was the control group and B was the experimental group in "A vs. $B$ ") to analyze the gene expression variations (Figure 2 and Additional file 8). A comparison of $\mathrm{Br}$ and Bo showed that 1352 and 1282 DEGs were significantly up-regulated and down-regulated, respectively. By contrast, 2278 DEGs were down-regulated and 2391 DEGs were up-regulated in Br compared with $\mathrm{Bg}$.

Moreover, 2140 DEGs were down-regulated and 2437 DEGs were up-regulated in Bo compared with Bg. The number of DEGs in Bg compared with $\mathrm{Br} / \mathrm{Bo}$ was more than $\mathrm{Br}$ vs. Bo, which indicates that the $\mathrm{A}$ and $\mathrm{C}$ genomes of Brassica were closer than the B genome. Among the 20 most abundantly expressed genes that were up-regulated or down-regulated in the pair comparison of the three diploids (Additional file 8), Bra015187, Bra026992, Bra017452, Bra029372, Bra028406, Bra017112, Bra036352, Bra000377, and Bra016934 were up-regulated in $\mathrm{Bg}$ compared with $\mathrm{Br}$ /
Bo. Moreover, Bra023103, Bra011285, Bra014371, Bra031070, Bra028805, and Bra006083 were down-regulated in Bg compared with $\mathrm{Br} / \mathrm{Bo}$. Most DEGs between $\mathrm{Br}$ and Bo differed from those between $\mathrm{Br}$ and $\mathrm{Bg}$ as well as between Bo and $\mathrm{Bg}$. Figure $3 \mathrm{~A}$ shows the distribution of the genes commonly expressed in $\mathrm{Br}, \mathrm{Bg}$, and $\mathrm{Bo}$, and 8932 genes were co-expressed in the three diploid libraries apart from the DEGs. A second filter of expression differences (at least twofold or greater) was performed in the pairwise comparisons to reduce the total number of significant changes. This analysis revealed 6401 significantly expressed genes, such as $\mathrm{Br}$ vs. $\mathrm{Bg}=4669, \mathrm{Br}$ vs. $\mathrm{Bo}=2634$, and $\mathrm{Bg}$ vs. $\mathrm{Bo}=4577$ (Figure 3B). The functional significance of the genes expressed in each library was examined, and the GO analysis results are shown in Figure 3C. The well-annotated gene sequences were assigned to 33 functional groups of the three main GO categories (cellular component, molecular function, and biological process). The results showed

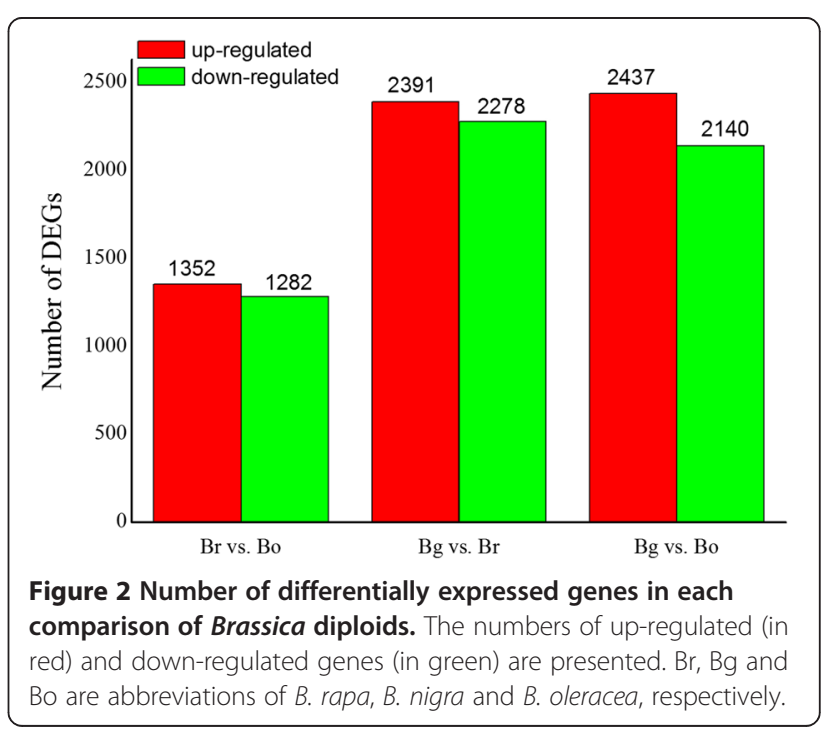


A

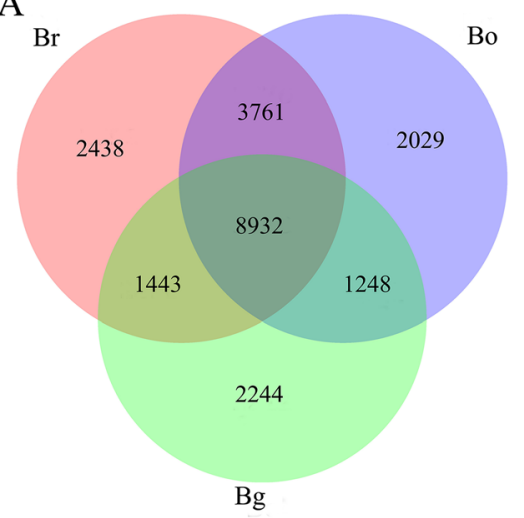

$\mathrm{C}$

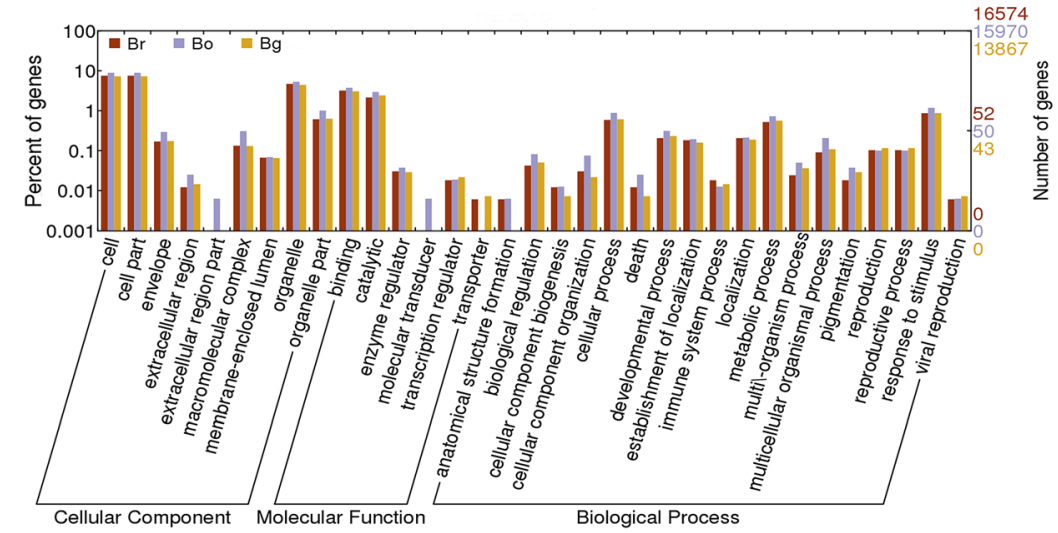

Figure 3 Distribution of expressed mRNAs in Brassica diploids. A. Venn diagram of genes expressed in Br, Bg and Bo. B. Venn diagram of unique expressed genes between pairwise of $\mathrm{Br}, \mathrm{Bg}$ and $\mathrm{Bo}$. C. GO classification of genes in Br, Bg and Bo.

that DGEs were primarily involved in the cell and organelle, in the binding, catalytic, cellular, and metabolic process, as well as in response to stimulus. Two specific processes, the extracellular region part and the molecular transducer, were unique to Bo. However, Bo lacked a transporter, and Bg lacked anatomical structure formation.

\section{DEGs among allopolyploids and ancestral diploid progenitors}

To identify the DEGs in allopolyploids and ancient diploid progenitors, the DGE profiles of $\mathrm{Br}$ vs. $\mathrm{Bn}$, Bo vs. $B n, B r$ vs. $B j, B g$ vs. $B j, B g$ vs. $B c$, and $B o$ vs. $B c$ were compared to analyze the gene expression variations after natural polyploidization (Figure 4 and Additional file 8). The results showed that 1230 DEGs were up-regulated and 324 DEGs were down-regulated in Bn compared with $\mathrm{Br}$, whereas 1872 DEGs were up-regulated and 797 DEGs were down-regulated in Bn compared with Bo. After natural polyploidization, 1519 DEGs were induced in $\mathrm{Bj}$ compared with $\mathrm{Br}$, whereas 508 DEGs were downregulated. Moreover, 2692 DEGs were induced in $\mathrm{Bj}$ compared with Bg, whereas 1393 DEGs were downregulated. With regard to Bc, 2099 DEGs were up-

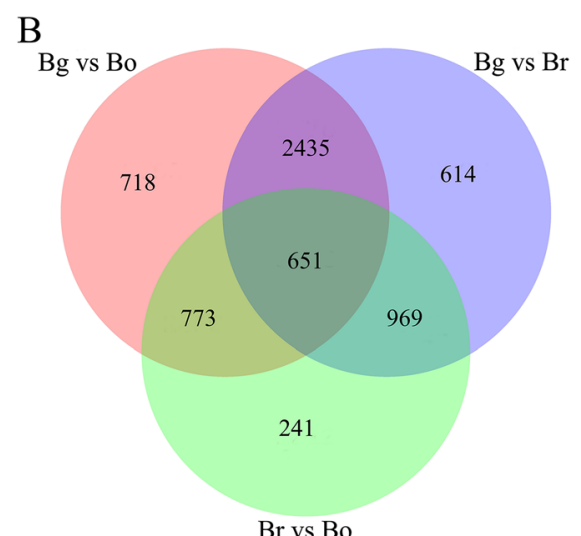

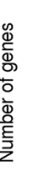

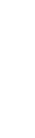

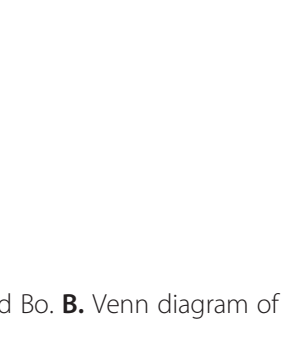

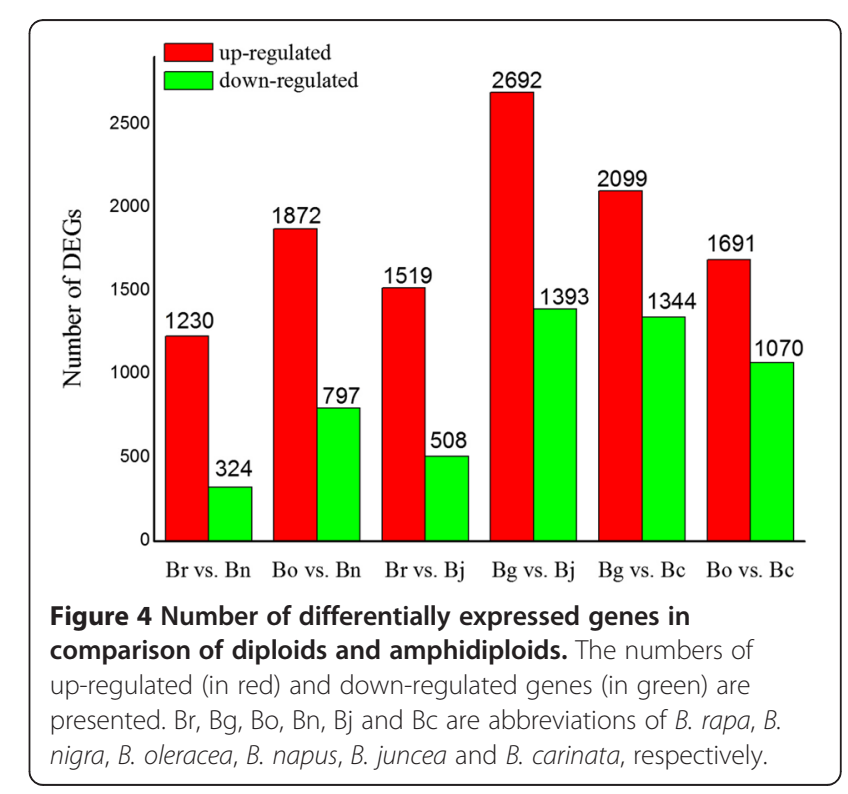


regulated and 1344 were down-regulated compared with $\mathrm{Bg}$, and 1691 DEGs were up-regulated and 1070 were down-regulated compared with $\mathrm{Bg}$. The variations in the gene expression among the diploids and amphidiploids are essential to the diversity of phenotype, growth, and production. The 20 most abundantly expressed genes that were up-regulated or down-regulated in the pair comparison of amphidiploids and diploids are listed in Additional file 8. The distribution of the genes that were commonly and uniquely expressed in amphidiploid and its ancestral diploids is shown in Figure 5. The results further show that 11810 genes were conserved in $\mathrm{Br}, \mathrm{Bo}$, and $\mathrm{Bn}$, whereas 1362,1666 , and 1824 genes were specifically expressed in $\mathrm{Br}, \mathrm{Bo}$, and $\mathrm{Bn}$, respectively (Figure $5 \mathrm{~A}$ ). A similar pattern was observed when $\mathrm{Bj}$ was compared with $\mathrm{Br} / \mathrm{Bg}$ (Figure $5 \mathrm{~B}$ ) and $\mathrm{Bc}$ with $\mathrm{Bg} / \mathrm{Bo}$ (Figure $5 \mathrm{C}$ ). The expressional differences of species-specific genes might be caused by the genome interaction during natural polyploidization. The GO pattern of the genes in amphidiploid and ancestral diploids is shown in Figure 6. Based on Figure $6 \mathrm{~A}$, the numbers of DGEs in the envelope, extracellular region, macromolecular complex, biological regulation, cellular component biogenesis, death, multicellular organism process, and pigmentation were different in $\mathrm{Br}, \mathrm{Bo}$, and $\mathrm{Bn}$. GOs of molecular transducer was found in Bo only. Apparent GO difference was observed among $\mathrm{Br}, \mathrm{Bg}$, and $\mathrm{Bj}$ (Figure 6C). As shown in Figure 6C, GOs of transporter were found in $\mathrm{Bg}$ only, and anatomical structure formation was not present in Bg.

\section{Functional annotation of DEGs}

Pathway enrichment analysis was performed on the expressed transcripts of the six DGE libraries. This analysis was performed by mapping all annotated genes in the KEGG database to search for significantly enriched genes involved in the metabolic or signal transduction pathways (Additional file 9). Among the genes with KEGG annotation, DEGs were identified in Bn compared with $\mathrm{Br}$.
In total, 894 DEGs were assigned to 109 KEGG pathways, and 13 of these pathways were significantly enriched with $Q$ values $\leq 0.05$ (red border region). The enriched pathways include metabolic, biosynthesis of secondary metabolites, and peroxisome. A similiar pathway enrichment was discovered in pair comparison of other libraries (Bo vs. $\mathrm{Bn}, \mathrm{Br}$ vs. $\mathrm{Bj}, \mathrm{Bg}$ vs. $\mathrm{Bj}, \mathrm{Bg}$ vs. $\mathrm{Bc}$, and Bo vs. $\mathrm{Bc}$ ). The 1562 DEGs identified in Bn vs. Bo were assigned to 122 KEGG pathways, 15 of which were significantly enriched. The 1171 DEGs identified in $\mathrm{Bj}$ vs. Br were assigned to 116 KEGG pathways, the 2373 DEGs identified in $\mathrm{Bj}$ vs. Bg were assigned to 121 pathways, the 1975 DEGs identified in Bc vs. Bg were assigned to 120 pathways, and the 1639 DEGs identified in Bc vs. Bo were assigned to 117 pathways. All these pathways are involved in the process of plant growth and stress reaction, which are important for the morphological and physiological differences among the Brassica species. The biosynthesis of unsaturated fatty acids, which was significantly enriched in Bo vs. Bn and $\mathrm{Bg}$ vs. Bc, explains the different fatty acid compositions in Brassica species [35,36]. The DEGs identified in the peroxisome pathway were related to the improved stress ability of Bn and Bj.

\section{Clustering of DEGs}

Hierarchical cluster analysis of the DEGs in $\mathrm{Br}, \mathrm{Bg}$, Bo, $\mathrm{Bn}, \mathrm{Bj}$, and $\mathrm{Bc}$ were performed to examine the similarity and diversity of gene expression (Additional file 4). All results were displayed by Java Treeview, where red and green represent the positive and negative values of gene expression, respectively. Generally, 651 genes with differential expression in $\mathrm{Br}, \mathrm{Bg}$, and $\mathrm{Bo}$ were clustered as DEG intersections (Figure 7A, Additional file 10). The comparison of $\mathrm{Br}, \mathrm{Bg}$, and Bo showed that 5417 DEGs were clustered as the union of DEGs (Additional file 11). Moreover, 285 DEGs in $\mathrm{Bn}, \mathrm{Br}$, and Bo were also clustered (Figure 7B and Additional file 9), and 3786 DEGs differentially expressed in $\mathrm{Bn}$ and $\mathrm{Br} / \mathrm{Bo}$ are listed in
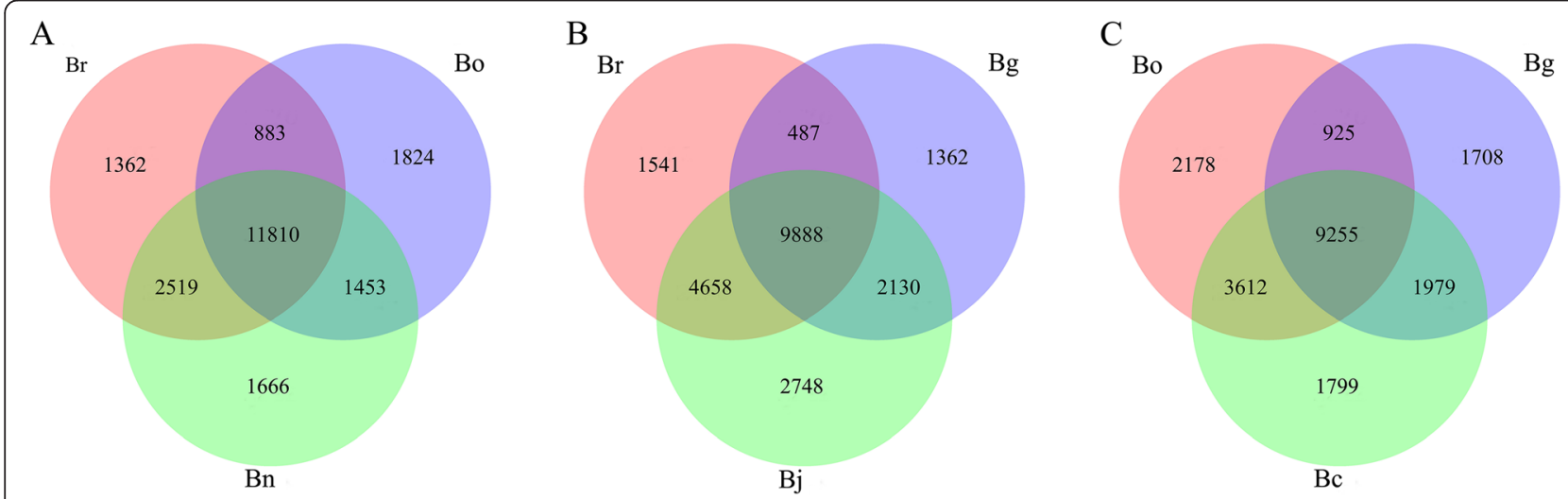

Figure 5 Distribution of the genes commonly and specifically expressed in diploids and amphidiploids. A. Venn diagram of genes expressed in $\mathrm{Br}, \mathrm{Bo}$ and $\mathrm{Bn}$. B. Venn diagram of genes expressed in $\mathrm{Br}, \mathrm{Bg}$ and $\mathrm{Bj}$. C. Venn diagram of genes expressed in Bg, Bo and BC. 

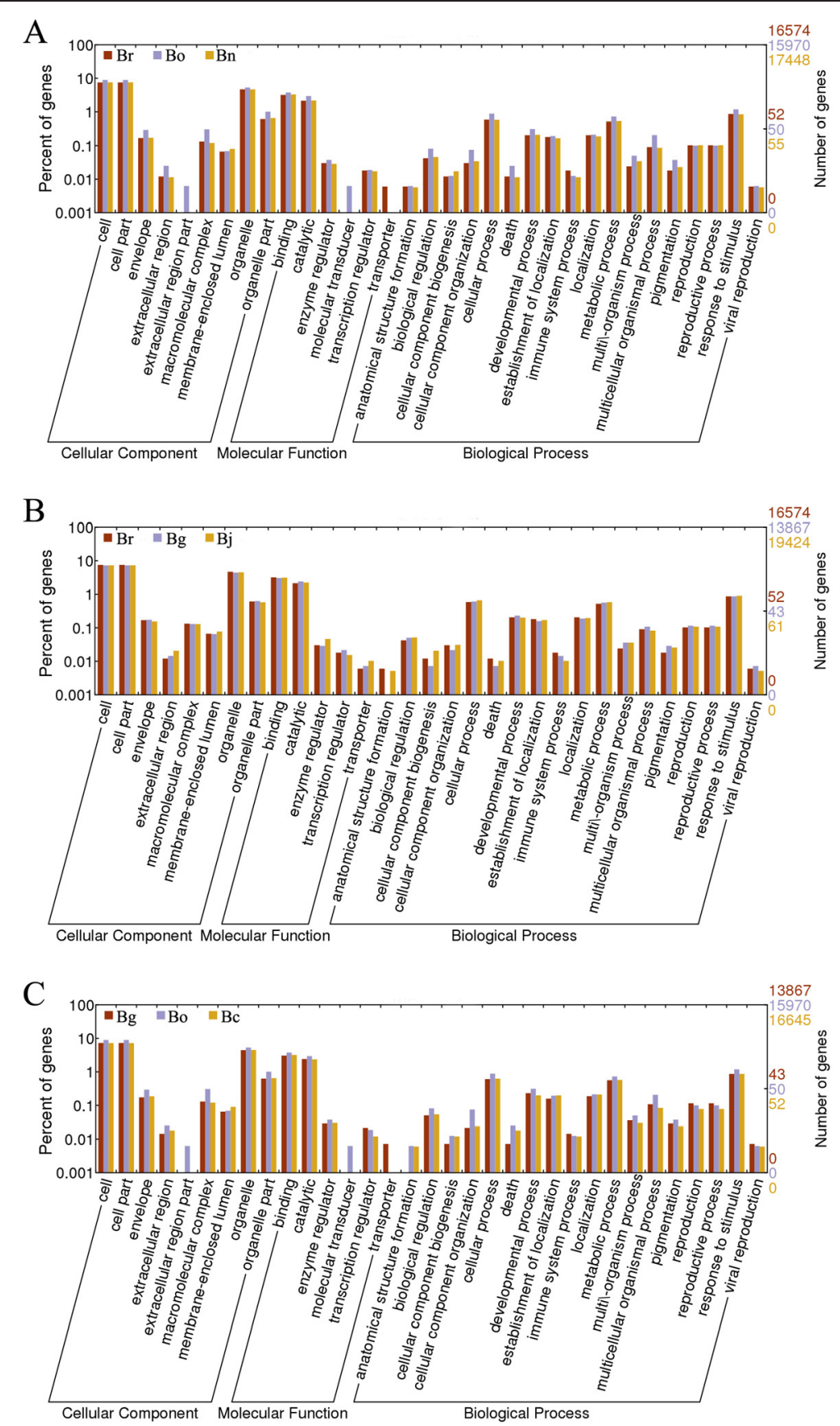

Figure 6 GO classification of genes in diploids and amphidiploids. A. GO classification of genes expressed in Br, Bo and Bn. B. GO classification of genes expressed in $\mathrm{Br}, \mathrm{Bg}$ and $\mathrm{Bj}$. C. $\mathrm{GO}$ classification of genes expressed in $\mathrm{Bg}, \mathrm{Bo}$ and $\mathrm{BC}$.

Additional file 11. The $630 \mathrm{DEGs}$ in $\mathrm{Bj}, \mathrm{Br}$, and $\mathrm{Bg}$ were also clustered (Figure 7C and Additional file 9), and 5590 DEGs differentially expressed in $\mathrm{Bj}$ and $\mathrm{Br} / \mathrm{Bg}$ are listed in Additional file 11. In addition, 726 DEGs in Bc, $\mathrm{Bg}$, and Bo were clustered (Figure 7D and Additional file 9), and 5264. DEGs differentially expressed in Bc and $\mathrm{Bg} / \mathrm{Bo}$ are listed in Additional file 11. The functional categories of these DEGs showed similar enrichment patterns for each comparison, including categories of metabolism, development, cellular transport, and interaction with the environment (data not shown). Genes with binding function were enriched in each comparison, which is different from previous reports $[32,33]$.

\section{Analysis of methyltransferase genes differentially expressed in Brassica}

Epigenetic variation has an important function in the evolution of plants. DNA methylation is included in this 


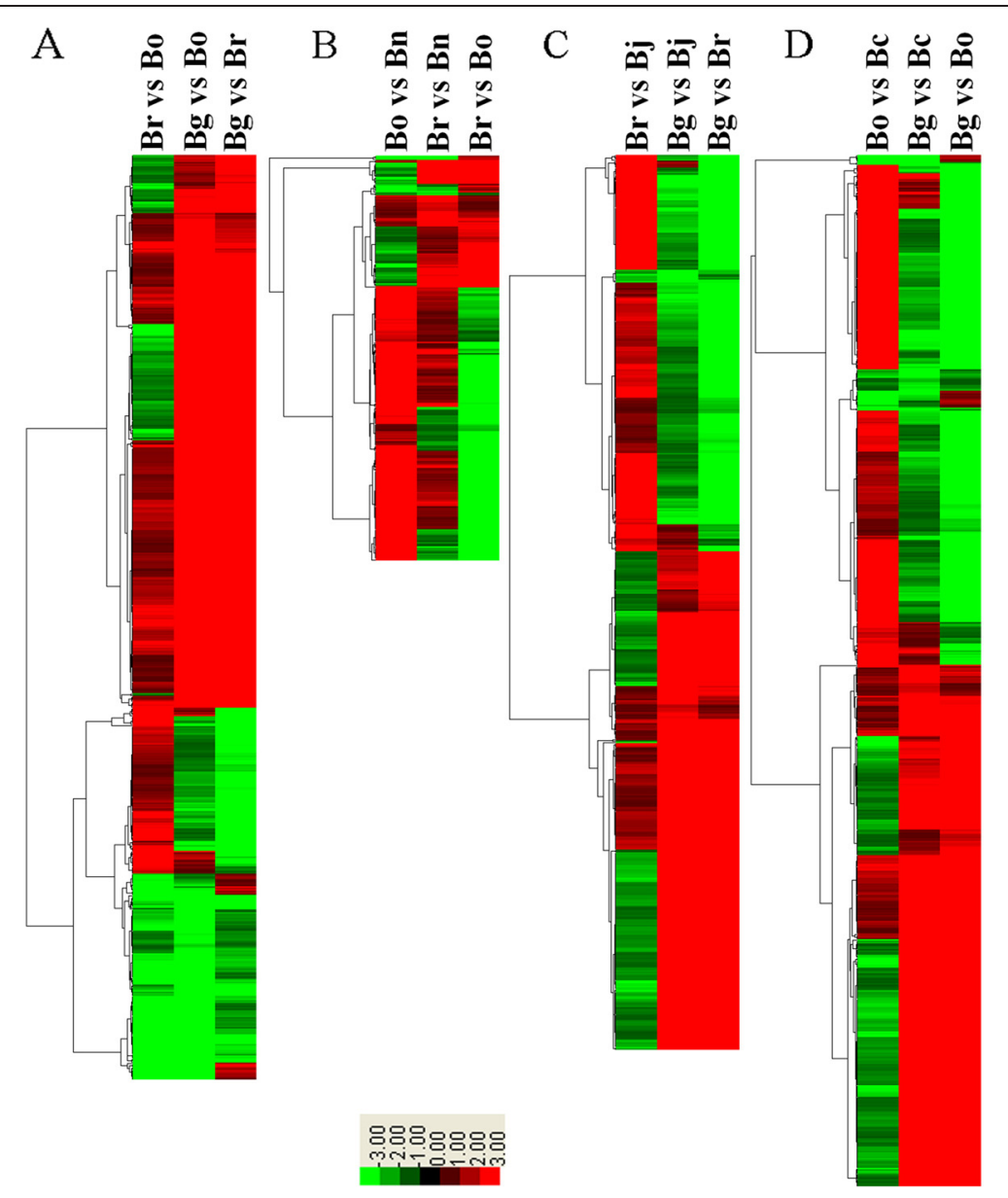

Figure 7 Hierarchical cluster analysis of differentially expressed transcripts. A. Clustering of genes expressed in diploids of Brassica. B. Clustering of genes expressed in $\mathrm{Br}, \mathrm{Bo}$ and $\mathrm{Bn}$. C. Clustering of genes expressed in Br, Bg and Bj. D. Clustering of genes expressed in Bg, $\mathrm{Bo}$ and $\mathrm{BC}$.

variation and has received much attention in previous years. Proteins such as methyltransferase are considered important for plant methylation [37,38]. Thus, the putative methyltransferase and methylase genes from all DEGs in each comparison were filtered to understand the mechanism of the changes in DNA methylation in Brassica (Additional file 12). Two methyltransferase genes (Bra003928 and Bra020452) were differentially expressed in $\mathrm{Br}, \mathrm{Bg}$, and $\mathrm{Bo}$, and 30 genes exhibited differential expression in $\mathrm{Br}$ vs. $\mathrm{Bo} / \mathrm{Bg}$ vs. $\mathrm{Bo} / \mathrm{Bg}$ vs. $\mathrm{Br}$. One methyltransferase gene (Bra008507) was differentially expressed in $\mathrm{Bn}, \mathrm{Br}$, and $\mathrm{Bo}$, and 23 genes exhibited differential expression in $\mathrm{Br}$ vs. $\mathrm{Bn} / \mathrm{Bo}$ vs. $\mathrm{Bn} / \mathrm{Br}$ vs. Bo. Five methyltransferase genes (Bra003396, Bra004391, Bra010977, Bra022603, and Bra024271) were differentially expressed in $\mathrm{Bj}, \mathrm{Br}$, and $\mathrm{Bg}$, and 36 genes exhibited differential expression in $\mathrm{Br}$ vs. $\mathrm{Bj} / \mathrm{Bg}$ vs. $\mathrm{Bj} / \mathrm{Bg}$ vs. $\mathrm{Br}$. Three methyltransferase genes (Bra003928, Bra004391, and Bra012494) were differentially expressed in Bc, Bg, and Bo, and 33 genes exhibited differential expression in
$\mathrm{Bg}$ vs. $\mathrm{Bc} / \mathrm{Bo}$ vs. $\mathrm{Bc} / \mathrm{Bg}$ vs. Bo. The results showed that Bra003928 was significantly down-regulated in $\mathrm{Br}$ compared with $\mathrm{Bg} / \mathrm{Bo}$, which was up-regulated in $\mathrm{Bn}$ compared with $\mathrm{Br}$ and down-regulated in $\mathrm{Bn}$ compared with Bo. The expression of Bra003928 in Bj was higher than in $\mathrm{Br}$ and lower than in Bg. The expression of this methyltransferase gene in $\mathrm{Bc}$ was significantly up-regulated than in Bg and Bo. Moreover, Bra020452 was obviously down-regulated in Bo compared with $\mathrm{Br} / \mathrm{Bg}$. Different expression values were also examined in Brassica amphidiploids compared with their ancestral diploid parents. The methyltransferase gene was up-regulated in $\mathrm{Bn}$ compared with $\mathrm{Br}$ and $\mathrm{Bo}$, which was also up-regulated in $\mathrm{BC}$ compared with $\mathrm{Bg}$ and $\mathrm{Bo}$. However, the expression value of $\mathrm{Bra} 020452$ in $\mathrm{Bj}$ was similar to that of $\mathrm{Br}$ and Bg.

\section{Non-additive genes expressed in Brassica amphidiploids}

The expression value of genes in Brassica amphidiploids ( $\mathrm{Bn}, \mathrm{Bj}$, and $\mathrm{Bc}$ ) were compared with the relative mid-parent 
value (MPV) to identify the genes with differential expression pattern. Up to 19844 genes in Bn showed differences in expression from MPV, 9605 (48.4\%) of these genes showed higher expression levels, whereas 10239 (51.6\%) showed lower expressions than MPV. Among the non-additively expressed genes, 9519 (48\%) genes were expressed at higher levels, whereas 10325 (52\%) genes were expressed at lower levels in $\mathrm{Br}$ than in Bo (Table 2). This finding is similar to the data reported by Jiang et al. (2013) [32]. With regard to $\mathrm{Bj}, 20317$ genes showed differences in expression from MPV, 11173 (55\%) of these genes were expressed higher in $\mathrm{Br}$ than in $\mathrm{Bg}$, and 9144 (45\%) genes were expressed at lower levels. Moreover, 19921 genes in Bc showed differences in expression from MPV, 8189 (46.1\%) of them were expressed higher in Bg than in Bo, whereas 10732 (53.9\%) genes were expressed lower. Significantly more non-additive genes than additive genes in amphidiploids implied the complicated evolution history of Brassica. In this study, no standard RNA sample was included in library construction. Given that two samples often differ in the total number of transcripts per cell, the differences in transcriptome size, not just the differences in RNA yields during extraction, can introduce biases [39-41]. In addition, polyploidization of plant species is a complicated process that is unequal to simple duplication or combination of ancient genomes; fractionation of duplicated genes would result in both gene and genome preferences in stabilized Brassica polyploids [5]. The challenge to RNA-seq is that the transcripts of duplicated genes are difficult to precisely assign to homologous polyploids, especially in the absence of a reference genome [42]. MPV is a valid criterion to assess non-additive gene expression changes and functional plasticity in allopolyploids [43]. For RNA-seq, another shortcoming is that many short reads likely align to identical reference sequences, which may be excluded from transcript counting, thereby affecting the accuracy of estimated gene expression level [42]. Given the information mentioned above, both the DGE and non- additive genes identified in this study might not be as accurate as expected, and thus further verification is necessary.

\section{Discussion}

Differences in gene expression among Brassica diploids Global Brassica research programs aim to explore valuable information on novel traits and to create stable lines. $\mathrm{Br}, \mathrm{Bg}$, and Bo exhibit many valuable agronomic traits including resistance against diseases and stress. These Brassica diploids have been suggested to have a triplication history [3]. Based on the DGE data of diploid Brassica species, multiple genes exhibited different expressional patterns in $\mathrm{Br}, \mathrm{Bg}$, and Bo. Moreover, 8932 genes were expressed in the leaf tissue of all three diploids. In addition, 2438, 2244, and 2029 genes were uniquely expressed in $\mathrm{Br}, \mathrm{Bg}$, and $\mathrm{Bo}$, respectively. However, 5417 DEGs were differently expressed among Brassica diploids including genes that encode proteins with binding function, transmembrane transporter, glycosyltransferase (Bra013229 and Bra016237), acyltransferase (Bra018329, Bra018412, Bra033107, Bra037338, and Bra037725), and methyltransferase (Bra036774, Bra003928, Bra005371, Bra018386, and Bra021673). Different copies of homologs in A, B, and C Brassica genomes and a comparative mapping of Brassica have revealed extensive differences among the A, B, and C genomes $[15,44]$. The transcriptome changes in Brassica diploids are possibly due to the polyploid history during species formation. These changes cause different genome dosages and sub-functionalization/neofunctionalization of genes, as well as morphological/physiological differences in $\mathrm{Br}, \mathrm{Bg}$, and Bo. This result would facilitate the gene exploration related to species-specific characters, thereby accelerating the breeding of Brassica.

\section{Gene expression differences among allopolyploids and ancestral diploid progenitors}

The expression differences in allotetraploids and diploids were analyzed by comparing the normalized value of

Table 2 Number of non-additively expressed genes in Brassica amphidiploids

\begin{tabular}{|c|c|c|c|c|c|c|c|c|}
\hline & \multirow{2}{*}{\multicolumn{2}{|c|}{$\begin{array}{l}\text { a } \% \\
\text { No. of non-additively expressed } \\
\text { genes Amphidiploid versus MPV }\end{array}$}} & b & $\%$ & $\mathrm{~b} / \mathrm{a}(\%)$ & c & $\%$ & $\mathrm{c} / \mathrm{a}(\%)$ \\
\hline & & & \multicolumn{3}{|c|}{$\begin{array}{l}\text { No. of non-additively expressed } \\
\text { genes Amphidiploid > MPV }\end{array}$} & \multicolumn{3}{|c|}{$\begin{array}{l}\text { No. of non-additively expressed } \\
\text { genes Amphidiploid < MPV }\end{array}$} \\
\hline $\mathrm{Bn}$ & 19844 & & 9605 & & 48.4 & 10239 & & 51.6 \\
\hline $\mathrm{Br}>\mathrm{Bo}$ & 9519 & 48 & 5220 & 54.3 & 54.8 & 4299 & 42 & 45.2 \\
\hline $\mathrm{Br}<\mathrm{Bo}$ & 10325 & 52 & 4385 & 45.7 & 42.5 & 5940 & 58 & 57.5 \\
\hline Bj & 20317 & & 10240 & & 50.4 & 10077 & & 49.6 \\
\hline $\mathrm{Br}>\mathrm{Bg}$ & 11173 & 55 & 6429 & 62.8 & 57.5 & 4744 & 47.1 & 42.5 \\
\hline $\mathrm{Br}<\mathrm{Bg}$ & 9144 & 45 & 3811 & 37.2 & 41.7 & 5333 & 52.9 & 58.3 \\
\hline$B C$ & 19921 & & 7990 & & 40 & 11931 & & 60 \\
\hline $\mathrm{Bg}>\mathrm{Bo}$ & 9189 & 46.1 & 3399 & 42.5 & 37 & 5790 & 48.5 & 63 \\
\hline $\mathrm{Bg}<\mathrm{Bo}$ & 10732 & 53.9 & 4591 & 57.5 & 42.8 & 6141 & 51.5 & 57.2 \\
\hline
\end{tabular}


genes expressed in each Brassica species. The results indicated that a larger number of gene expressional differences were identified between allotetraploids and diploids than among diploids. Although 11810 genes were conserved in $\mathrm{Bn}, \mathrm{Br}$, and $\mathrm{Bo}, 3102$ DEGs were upregulated in $\mathrm{Bn}$ compared with $\mathrm{Br}$ or $\mathrm{Bo}$, and 1121 DEGs were down-regulated in $\mathrm{Bn}$ after natural polyploidization. Similarly, DEGs were also analyzed in $\mathrm{Bj}$ and $\mathrm{BC}$ after polyploidization, and gene expressional changes were considered with parental preference. Zhao et al. (2013) also found that the gene expression in Brassica hexaploid is more similar to $\mathrm{Br}$ than to $\mathrm{Bc}$ [33]. In accordance with previous results, a large number of DEGs in natural $\mathrm{Bn}$ and $\mathrm{Br} / \mathrm{Bo}$ suggests that the gene expression differences in resynthesized $\mathrm{Bn}$ might be effectively inherited after polyploidization $[32,45,46]$. These results indicated that long-term and immediate responses to polyploidization are complicated. Genome-biased expression and silencing of genes are also observed in natural and synthetic cotton [47]. Zhao et al. (2013) suggested that this observation might be due to the interactions of cytoplasm and nuclear genome during polyploidization [33]. Hitherto, $B j$ and $B c$ have been used for the creation of synthesized Brassica allopolyploids (AABBCC, $\mathrm{AABC}, \mathrm{BBAC}$, and $\mathrm{CCAB}$ ) [48]. However, polyploidization of $B j$ and $B c$ have not been thoroughly studied. Given that the B genome possesses valuable agronomic traits including black-leg resistance [49], the study of Bgenome evolution during the polyploidization of $\mathrm{Bj}$ and $\mathrm{BC}$ is meaningful to the exploration of B-genome desirable traits. In the present research, many gene expressional differences in $\mathrm{Bj}$ and $\mathrm{Bc}$ after polyploidization were explored. The results showed that 5590 genes were differentially expressed in $\mathrm{Bj}, \mathrm{Br}$, and $\mathrm{Bg}$, including genes that encode acyltransferase and metyltransferase. Moreover, the $\mathrm{DEGs}$ in $\mathrm{Bj}$ and $\mathrm{Bc}$ after polyploidization were more than that in $\mathrm{Bn}$, which is partially due to the lack of a reference genome in this research. The $\mathrm{B}$ genome is more diversified than the $\mathrm{A}$ and $\mathrm{C}$ genomes [48]; hence, some B genome-specific information were neglected during the analysis of DGE data. Most of the commonly expressed genes in the diploids were nonadditively expressed in allotetraploids, which is similar to the non-additive pattern in synthesized Bn and Arabidopsis $[32,49]$. The repression and activation of these genes in allotetraploids are responsible for the sub-functionalization of duplicated genes [47], which indicates a more complicated gene expression in allopolyploids rather than a simple combination of two genomes $[46,48]$. All of these non-additively expressed genes are important in studying the genome polyploidization of Brassica. The expressional changes in allotetraploids are necessary for the adjustment to the environment during natural polyploidization [33].

\section{Putative methyltransferase genes in Brassica allotetraploids}

One of the epigenetic variations is DNA methylation, which is important to genome activity. Plant polyploidization is normally accompanied with various phenotypic changes that are partially induced by new methylation changes during the interaction of different genomes [50]. Extensive DNA methylation differences have been reported in synthesized Bn [45,51]. In the present research, 23,36 , and 33 methyltransferase genes were differentially expressed after the polyploidization of $\mathrm{Bn}, \mathrm{Bj}$, and $\mathrm{Bc}$, respectively. The methyltransferase gene Bra020452 was up-regulated in Bn compared with $\mathrm{Br}$ and Bo, whereas the expression value of this gene in the early generations of resynthesized $\mathrm{Bn}$ was lower than that of natural $\mathrm{Bn}$ [32]. This finding implies the complexity of gene activation and silencing mechanism during Brassica polyploidization. Whether these methylation changes in Brassica are responsible for the different expressions of DEGs in allotetraploids needs to be verified. Further research of these genes is important to comprehend the transcriptome changes during Brasssica polyploidization.

\section{Conclusions}

The genus Brassica includes a group of crops with important economic and nutritional values, and these crops are most closely related to Arabidopsis. Brassica and Arabidopsis have been confirmed to originate from a putative hexaploid ancestor. Triplication occurred after the divergence of Brassica and Arabidopsis to form a genomic complexity of Brassica [3]. Three allopolyploids, which arose from the natural hybridization of A, B, and $\mathrm{C}$ genomes, introduced extensive genome reshuffling and gene loss, as well as neo- and sub-functionalization of duplicate genes [6]. Therefore, the Brassica species are taken as an important model for the evolution of polyploids. Unfortunately, acknowledging the ancestors of Brassica polyploids is difficult, and these tetraploids are suspected to have multiple origins [52]. Resynthesizing Brassica allopolyploids have provided an alternative way to study polyploidization, but the research in this area mainly focused on B. napus [32]. An overview of the transcriptome differences among natural Brassica species would be interesting to gain initial knowledge on species diversification and polyploidization. This study demonstrated the DGE approach in characterizing the transcriptome of Brassica diploids and allotetraploids. However, the sampling from each genotype of Brassica may not capture the true range of phenotypes that exists within this genus. The DEGs during the evolution of Brassica diploids from a common hexaploid ancestor with Arabidopsis were revealed. Moreover, the DEGs in the natural polyploidization of Brassica allotetraploids from the hybridization of diploids were determined. 
Future work should concentrate on the function analysis of these DEGs, particularly stress resistance and methylase genes. Analysis should be performed to uncover the genetic and epigenetic mechanisms that would result in the phenotypic and physiologic differences among Brassica species. Elucidation of these differences is important to the discovery and utilization of genes for Brassica breeding and to shed light on Brassica evolution.

\section{Methods}

\section{Plant materials}

Diploid species $B$. rapa cv. Aikangqing (AA, $2 n=20$ ), $B$. nigra $\mathrm{cv}$. Marathi $(\mathrm{BB}, 2 n=16)$, and $B$. oleracea $\mathrm{cv}$. Zhonghua Jielan $(C C, 2 n=18)$ were used in the experiment. Amphidiploids B. napus cv. Yangyou 6 (AACC, $2 n=38$ ), B. juncea cv. Luzhousileng (AABB, $2 n=36$ ), and B. carinata cv. Dodolla (BBCC, $2 n=34)$ were also used as experimental materials. Plant materials were prepared and collected according to the procedures described by Kong et al. (2011) and Jiang et al. (2013) $[32,53]$. All plants were cultivated in climate chambers at $25^{\circ} \mathrm{C}, 16 \mathrm{~h} \mathrm{light} / 8 \mathrm{~h}$ dark photoperiod, and $70 \%$ relative humidity. The first true leaves from the three plants of each genotype were pooled at the same physiologic stage (28-day-old plants) and frozen at $80^{\circ} \mathrm{C}$ prior to use.

\section{RNA preparation, illumina RNA-sequencing, and data processing}

Total RNA was extracted from the leaves by using an RNAiso Plus (Takara) according to the manufacturer's protocol. RNA concentrations were measured using a Qubit fluorometer, and the integrity was confirmed using a 2100 Bioanalyzer (Agilent Technologies). DGE libraries were prepared using an Illumina Gene Expression Sample Prep Kit, and NlaIII and MmeI were used for tag preparation. Single-chain molecules were fixed onto a Solexa sequencing chip (flowcell) and sequenced by an Illumina HiSeq 2000 System. Millions of raw 35 bp sequences were generated. Image analysis, base calling, generation of raw tags, and counting of tags were performed using the Illumina pipeline [34]. Empty tags (no tag sequence between the adaptors), adaptors, lowquality tags (tags containing one or more unknown nucleotides " $\mathrm{N}$ "), and tags with a copy number of 1 were removed from the raw sequences to obtain clean tags (21 bp) that contain CATG.

\section{Mapping of reads to the reference sequence}

To identify the gene expression patterns in each genotype of Brassica, all clean tags were annotated by mapping onto the B. rapa genome [12] by using the SOAP2 software, and a maximum of one nucleotide mismatch is allowed [54]. All tags mapped to reference sequences were filtered, and the remaining tags were designated as ambiguous tags. Mapping events on sense and antisense sequences were included in the data processing. For gene expression analysis, the number of expressed tags was calculated and then normalized to the number of transcripts per million tags (TPM) [34,55]. The DEGs were screened and used for mapping and annotation [56,57]. Gene annotation was conducted using Blast2GO [58]. When the gene ontology (GO) database was searched, the GO categorization of all DEGs was displayed as three hierarchies for cellular component, molecular function, and biological process. Web gene ontology annotation plot was used to classify the genes mapped by each DGE library [59]. Clustering analysis of differential gene expression pattern was also conducted using a hierarchical clustering explorer $[60,61]$. In the present study, statistical comparison of the gene expression was performed according to the script written by Audic and Claverie [56]. False discovery rate $(F D R) \leq 0.001$ and $\log 2$ ratio $\geq 1$ were used as threshold to judge significance of gene expression difference [57]. Pathway enrichment analysis of differential gene expression was conducted to understand further the gene function through blasting the KEGG database. A $P$-value of 0.05 was selected as the threshold for considering a gene set as significantly enriched.

\section{Availability of supporting data}

The sequence datasets that support the results of this article have been deposited in the Gene Expression Omnibus (GEO) at NCBI and are accessible under the accession GSE43246 (http://www.ncbi.nlm.nih.gov/geo/ query/acc.cgi?acc=GSE43246).

\section{Additional files}

Additional file 1: Distribution of total clean tags and distinct clean tags over different tag abundance categories in each DGE library.

(A) Distribution of total tags. Numbers in the brackets of indicate the range of copy numbers for a specific category of tags. For example, $[2,5]$ means all the tags in this category has 2 to 5 copies. Numbers in the parentheses show the total tag copy number for all the tags in that category. (B) Distribution of distinct tags. Numbers in the square brackets indicate the range of copy numbers for a specific category of tags. Numbers in the parentheses show the total types of tags in that category.

Additional file 2: Summary of tag mapping in DGE analysis for six libraries.

Additional file 3: Mapping results of total tags and distinct tags of species in six libraries. Normalized tag copy number was calculated by dividing tag counts for each gene with the total number of tags generated for each library and are presented per one million transcripts. $\mathrm{PM}$ and $1 \mathrm{MM}$ stand for perfect match and 1 miss match, respectively. (A) Mapping of total tags. (B) Mapping of distinct tags.

Additional file 4: List of all $B$. rapa genes identified by DGE. The first column represents names of the identified genes. Br_sense_raw and Br_antisense_raw mean the number of tags mapped to sense and antisense genes identified in DGE library of B. rapa. Br_sense_norm and $\mathrm{Br}$ _antisense_norm mean total times of detected tags per million in DGE library of B. rapa. GO Component, GO Function and GO Process mean 
the three main categories (cellular component, molecular function and biological process) in the $\mathrm{GO}$ classification, respectively.

Additional file 5: List of all $A$. thaliana genes identified by DGE. The first column represents names of the identified Arabidopsis genes. Br_raw means the number of DGE tags in $B$. rapa which were mapped to $A$. thaliana genome. $\mathrm{Br}$ _nom means total times of detected tags per million in DGE library of B. rapa.

Additional file 6: Sequencing saturation analysis of the seven libraries of $B$. rapa $(\mathrm{Br}), B$. nigra $(\mathrm{Bg})$, B. oleracea (Bo), B. napus (Bn), B. juncea (Bj), B. carinata (BC). The number of detected genes was enhanced as the sequencing amount (total tag number) increased.

Additional file 7: Distribution of ratio of distinct tag copy number in comparison of diploids and amphidiploids. ' $A$ ' was the control and ' $B$ ' was experimental group in 'A vs. B'.

Additional file 8: List of differentially expressed genes and the top 20 most up-regulated and down-regulated genes between diploids and amphidiploids (' $A$ ' was the control and ' $B$ ' was experimental group in 'A vs. $\mathbf{B}$ '). TPM: transcript copies per million tags. Raw intensity: the total number of tags sequenced for each gene. FDR: false discovery rate. We used FDR $<0.001$ and the absolute value of $\log 2$ Ratio $\leq 1$ as the threshold to judge the significance of gene expression difference. In order to calculate the log2Ratio and FDR, we used TPM value of 0.001 instead of 0 for genes that do not express in one sample.

Additional file 9: List of genes for pathway enrichment analysis. Pathways with $Q$ value $\leq 0.05$ are significantly enriched in $D E G$, see red-border region ('A' was the control and ' $B$ ' was experimental group in 'A vs. B').

Additional file 10: List of intersection DEGs used for HCE clustering analysis.

Additional file 11: List of union DEGs used for HCE clustering analysis.

Additional file 12: List of putative methyltransferase genes differentially expressed in Brassica.

\section{Competing interests}

The authors declare that they have no competing interests.

\section{Authors' contributions}

YPW conceived and designed the study. JJ, YW, BZ and TF participated in the experiments. JJ and FY analyzed the data. All authors drafted the manuscript and approved the final manuscript.

\section{Acknowledgments}

This study was supported by the National Key Basic Research Program of China (2015CB150201), the NSFC projects (31330057,31401414), the Jiangsu Province Science Foundation (BK20140478, 14KJB210008), the Priority Academic Program Development of Jiangsu Higher Education Institutions, and the Innovation Team of Yangzhou University, China. We sincerely appreciated Prof. Dr. Rod Snowdon and Dr. Christian Obermeier for their helpful suggestions and discussions on the manuscript.

Received: 30 July 2014 Accepted: 8 January 2015

Published online: 27 January 2015

\section{References}

1. Leitch AR, Leitch IJ. Genomic plasticity and the diversity of polyploidy plants. Science. 2008;320:481-3.

2. U N. Genomic analysis in Brassica with specific reference to the experimental formation of $B$. napus and peculiar mode of fertilization. Jpn J Bot. 1935;7:389-452.

3. Lysak MA, Koch MA, Pecinka A, Schubert I. Chromosome triplication found across the tribe Brassiceae. Genome Res. 2005;15:516-25.

4. Koch MA, Haubold B, Mitchell-Olds T. Comparative evolutionary analysis of chalcone synthase and alcohol dehydragenase loci in Arabidopsis, Arabis, and related genera (Brassicaceae). Mol Biol Evol. 2000;17:1483-98.

5. Woodhouse MR, Cheng F, Pires JC, Lisch D, Freeling M, Wang XW. Origin, inheritance, and gene regulatory consequences of genome dominance in polyploids. Proc Natl Acad Sci U S A. 2014;111(1):5283-8.
6. Cheung F, Trick M, Drou N, Lim YP, Park JY, Kwon SJ, et al. Comparative analysis between homologous genome segments of Brassica napus and its progenitor species reveals extensive sequence-level divergence. Plant Cell. 2009:21:1912-28

7. Osborn TC. The contribution of polyploidy to variation in Brassica species. Physiol Plant. 2004;121:531-6.

8. He X, Zhang J. Rapid subfunctionalization accompanied by prolonged and substantial neofunctionalization in duplicate gene evolution. Genetics. 2005;169:1157-64.

9. Rana D, van den Boogaart T, O'Neill CM, Hynes L, Bent E, Macpherson L, et al. Conservation of the microstructure of genome segments in Brassica napus and its diploid relatives. Plant J. 2004;40:725-33.

10. Town CD, Cheung F, Maiti R, Crabtree J, Haas BJ, Wortman JR, et al. Comparative genomics of Brassica oleracea and Arabidopsis thaliana reveals gene loss, fragmentation and dispersal following polyploidy. Plant Cell. 2006;18:1348-59.

11. Yang TJ, Kim JS, Kwon SJ, Lim KB, Choi BS, Kim JA, et al. Sequence-level analysis of the diploidization process in the triplicated FLOWERING LOCUS C region of Brassica rapa. Plant Cell. 2006;18:1339-47.

12. Wang XW, Wang HZ, Wang J, Sun RF, Wu J, Liu SY, et al. The genome of mesopolyploid crop species Brassica rapa. Nat Genet. 2011;43:1035-9.

13. Parkin IAP, Gulden SM, Sharpe AG, Lukens L, Trick M, Osborn TC, et al. Segmental structure of the Brassica napus genome based on comparative analysis with Arabidopsis thaliana. Genetics. 2005;171:765-81.

14. Ramchiary N, Padmaja KL, Sharma S, Gupta V, Sodhi YS, Mukhopadhyay A, et al. Mapping of yield influencing QTL in Brassica juncea: implications for breeding of a major oilseed crop of dryland areas. Theor Appl Genet. 2007;115:807-17.

15. Panjabi P, Jagannath A, Bisht NC, Padmaja KL, Sharma S, Gupta V, et al. Comparative mapping of Brassica juncea and Arabidopsis thaliana using Intron Polymorphism (IP) markers: homoeologous relationships, diversification and evolution of the A, B and C Brassica genomes. BMC Genomics. 2008;9:113-31

16. Wang J, Lydiate DJ, Parkin IAP, Falentin C, Delourme R, Carion PWC, et al. Integration of linkage maps for the amphidiploid Brassica napus and comparative mapping with Arabidopsis and Brassica rapa. BMC Genomics. 2011;12:101-20.

17. Szadkowski E, Eber F, Huteau V, Lode M, Huneau C, Belcram H, et al. The first meiosis of resynthesized Brassica napus, a genome blender. New Phytol. 2010;186:102-12.

18. Gaeta RT, Pires JC. Homoeologous recombination in allopolyploids: the polyploidy ratchet. New Phytol. 2010;186:18-28.

19. Trick M, Cheung F, Drou N, Fraser F, Lobenhofer EK, Hurban P, et al. A newly-developed community microarray resource for transcriptome profiling in Brassica species enables the confirmation of Brassica-specific expressed sequences. BMC Plant Biol. 2009;9:50-9.

20. Edwards D, Batley J, Snowdon RJ. Accessing complex crop genomics with next-generation sequencing. Theor Appl Genet. 2013;126:1-11.

21. Schmutz J, Cannon SB, Schlueter J, Ma JX, Mitros T, Nelson W, et al. Genome sequence of the palaeopolyploid soybean. Nature. 2010;463:178-83.

22. The Potato Genome Sequencing Consortium. Genome sequence and analysis of the tuber crop potato. Nature. 2011;475:189-95.

23. The Tomato Genome Sequencing Consortium. The tomato genome sequence provides insights into fleshy fruit evolution. Nature. 2012;485:635-41.

24. Wang KB, Wang ZW, Li FG, Ye WW, Wang JY, Song GL, et al. The draft genome of a diploid cotton Gossypium raimondii. Nat Genet. 2012;44:1098-103.

25. Shan $X, L i Y$, Jiang $Y$, Jiang $Z$, Hao W, Yuan Y. Transcriptome profile analysis of maize seedling in response to high-salinity, drought and cold stresses by deep sequencing. Plant Mol Biol Rep. 2013;31:1485-91.

26. Mueller BSF, Sakamoto T, Silverira RDD, Zambussi-Carvalho PF, Pereira M, Pappas Jr GJ, et al. Differentially expressed genes during flowering and grain filling common bean (Phaseolus vulgaris) grown under drought stress conditions. Plant Mol Biol Rep. 2014;32:438-51.

27. Trick M, Long Y, Meng JL, Bancroft I. Single nucleotide polymorphysn (SNP) discovery in the polyploidy Brassica napus using Solexa transcriptome sequencing. Plant Biotechnol J. 2009;7:334-46.

28. Bancroft I, Morgan C, Fraser F, Higgins J, Wells R, Clissold L, et al. Dissecting the genome of the polyploidy crop oilseed rapa by transcriptome sequencing. Nat Biotechnol. 2011;29:762-6.

29. Higgins J, Magusin A, Trick M, Fraser F, Bancroft I. Use of mRNA-seq to discriminate contributions to the transcriptome from the constituent 
genome of the polyploidy crop species Brassica napus. BMC Genomics. 2012;13:247-60.

30. Renaud G, LaFave MC, Liang J, Wolfsberg TG, Burgess SM. trieFinder: an efficient program for annotating digital gene expression (DGE) tags. BMC Bioinformatics. 2014;15:329-35.

31. Gaeta RT, Yoo SY, Pires JC, Doerge RW, Chen ZJ, Osborn TC. Analysis of gene expression in resynthesized Brassica napus allopolyploids using Arabidopsis 70mer oligo microarrays. Plos One. 2009:4(3):e4760.

32. Jiang JJ, Shao YL, Du K, Ran LP, Fang XP, Wang YP. Use of digital gene expression to discriminate gene expression differences in early generations of resynthesized Brassica napus and its diploid progenitors. BMC Genomics. 2013;14:72-82

33. Zhao Q, Zou J, Meng JL, Mei SY, Wang JB. Tracing the transcriptome changes in synthetic trigenomic allohexaploids of Brassica using an RNA-seq approach. Plos One. 2013;8(7):e68883.

34. Hoen PAC, Ariyurek Y, Thygesen HH, Vreugdenhil E, Vossen RHAM, Menezes RX, et al. Deep sequencing-based expression analysis shows major advances in robustness, resolution and inter-lab portability over five microarray platforms. Nucl Acids Res. 2008;36:141-51.

35. Batista C, Barro L, Carvalho AM, Ferreira I. Nutritional and nutraceutical potential of rape (Brassica napus L. var. napus) and 'tronchuda' cabbage (Brassica oleracea L. var. costata) inflorescences. Food Chem Toxicol. 2011:49:1208-14

36. Vuorinen AL, Kalpio M, Linderborg KM, Kortesniemi M, Lehto K, Niemi J, et al. Coordinate changes in gene expression and triacylglycerol composition in the developing seeds of oilseed rape (Brassica napus) and turnip rape (Brassica rapa). Food Chem. 2014;145:664-73.

37. Bird A. Perceptions of epigenetics. Nature. 2007:447:396-8.

38. Chinnusamy V, Zhu JK. Epigenetic regulation of stress responses in plants. Curr Opin Plant Biol. 2009;12(2):133-9.

39. Loven J, Orlando DA, Sigova AA, Lin CY, Rahi PB, Burge CB, et al. Revisiting global gene expression analysis. Cell. 2012;151(3):476-82.

40. Coate JE, Doyle JJ. Quantifying whole transcriptome size, a prerequisite for understanding transcriptome evolution across species: an example from a plant allopolyploid. Genome Biol Evol. 2010;2:534-46.

41. Coate JE, Doyle JJ. Variation in transcriptome size: are we getting the message? Chromosoma. 2014; DOI 10.1007/s00412-014-0496-3.

42. Ilut DC, Coate JE, Luciano AK, Owens TG, May GD, Farmer A, et al. A comprehensive transcriptome study of an allotetraploid and its diploid progenitors illustrates the unique advantages and challenges of RNA-seq in plant species. Am J Bot. 2012;99(2):383-96.

43. Jackson S, Chen ZJ. Genomic and expression plasticity of polyploidy. Curr Opin Plant Biol. 2010;13:153-9.

44. Schranz ME, Lysak MA, Mitchell-Olds T. The ABC's of comparative genomics in the Brassicaceae: building blocks of crucifer genomes. Trends Plant Sci. 2006;11:535-42.

45. Xu YH, Zhong L, Wu XM, Fang XP, Wang JB. Rapid alterations of gene expression and cytosine methylation in newly synthesized Brassica napus allopolyploids. Planta. 2009;229:471-83.

46. Szadkowski E, Eber F, Huteau V, Lode M, Coriton O, Jenczewski E, et al. Polyploid formation pathways have an impact on genetic rearrangement in resynthesized Brassica napus. New Phytol. 2011;191:884-94.

47. Adams KLR, Gronn R, Percifield R, Wendel JF. Genes duplicated by polyploidy show unequal contributions to the transcriptome and organ-specific reciprocal silencing. Proc Natl Acad Sci U S A. 2003;100:4649-54.

48. Mason AS, Huteau V, Eber F, Coriton O, Yan GJ, Nelson MN, et al. Genome structure affects the rate of autosyndesis and allosyndesis in AABC, BBAC, CCAB Brassica interspecific hybrids. Chromosome Res. 2010;18:655-66.

49. Wang JL, Tian L, Lee HS, Wei NE, Jiang HM, Watson B, et al. Genome wide non-additive gene regulation in Arabidopsis allotetraploids. Genetics. 2006;172:507-17

50. Gaeta RT, Pires JC, Iniguez-Luy F, Leon E, Osborn TC. Genomic changes in resynthesized Brassica napus and their effect on gene expression and phenotype. Plant Cell. 2007;19:3403-17.

51. Lukens LN, Pires JC, Leon E, Vogelzang R, Oslach L, Osborn T. Patterns of sequence loss and cytosine methylation within a population of newly synthesized Brassica napus allopolyploids. Plant Physiol. 2006;140:336-48.

52. Flannery ML, Mitchell FJG, Coyne S, Kavanagh TA, Burke Jl, Salamin N, et al. Plastid genome characterization in Brassica and Brassicaceae using a new set of nine SSR. Theor Appl Genet. 2006;113:1221-31.
53. Kong F, Mao SJ, Jiang JJ, Wang J, Fang XP, Wang YP. Proteomic changes in newly synthesized Brassica napus allotetraploids and their early generations. Plant Mol Biol Rep. 2011;29:927-35.

54. Li RQ, Yu C, Li YR, Lam TW, Yiu SM, Kristiansen K, et al. SOAP2: an improved ultrafast tool for short read alignment. Bioinformatics. 2009;25:1966-7.

55. Morrissy AS, Morin RD, Delaney A, Zeng T, McDonald H, Jones S, et al. Next-generation tag sequencing for cancer gene expression profiling. Genome Res. 2009;19:1825-35.

56. Audic S, Claverie JM. The significance of digital gene expression profiles. Genome Res. 1997;7:986-95.

57. Benjamini BY, Yekutieli D. The control of the false discovery rate in multiple testing under dependency. Ann Stat. 2011;29:1165-88.

58. Conesa A, Gotz S, Garcia-Gomez JM, Terol J, Talon M, Robles M. Blast2GO: a universal tool for annotation, visualization and analysis in functional genomics research. Bioinformatics. 2005;21:3674-6.

59. Ye J, Fang L, Zheng H, Zhang Y, Chen J, Zhang Z, et al. WEGO: a web tool for plotting GO annotations. Nucl Acids Res. 2006;34:293-7.

60. Eisen MB, Spellman PT, Brown PO, Botstein D. Cluster analysis and display of genome-wide expression. Proc Natl Acad Sci U S A. 1998;95:14863-8.

61. Saldanha AJ. Java tree view-extensible visualization of microarray data. Bioinformatics. 2004;20:3246-8.

\section{Submit your next manuscript to BioMed Central and take full advantage of:}

- Convenient online submission

- Thorough peer review

- No space constraints or color figure charges

- Immediate publication on acceptance

- Inclusion in PubMed, CAS, Scopus and Google Scholar

- Research which is freely available for redistribution 